\title{
Kampf gegen die Unwissenschaft
}

LÁszló Honti (Chefred.), A nyelvrokonságról. Az török, sumer és egyéb áfium ellen való orvosság. [Über Sprachverwandtschaft. Ein Gegenmittel gegen türkische, sumerische und andere Irrlehren.] Budapest: Tinta Könyvkiadó, 2010. $371 \mathrm{~S}$.
Das vorliegende Werk richtet sich an Ungarn, die sich für die Geschichte ihrer Muttersprache interessieren. Es bietet einen kurzen Überblick über den wissenschaftlichen Hintergrund der Sprachverwandtschaft und widerlegt die zahlreichen unwissenschaftlichen Darstellungen 
der Vorgeschichte und des Ursprungs der Sprache, die vor allem in Ungarn vorgelegt wurden. In Ungarn wird die finnisch-ugrische Sprachverwandtschaft in der öffentlichen Debatte heute noch bestritten. Diese unwissenschaftlichen Auffassungen werden im vorliegenden Werk als alternative Theorien bezeichnet. Vorgestellt werden auch die revolutionären Auffassungen von der finnisch-ugrischen Urgeschichte, die einige Finnen, Esten und Ungarn vorgebracht haben. Alle beteiligten Autoren sind namhafte Vertreter der Finnougristik oder der allgemeinen Sprachwissenschaft. Den vorgeschichtlichen Aspekt beleuchtet István Fodor.

Der Titel des Buchs enthält einen intertextuellen Verweis auf Miklós Zrínyis bekanntes Werk $A z$ török áfium ellen való orvosság, eine gegen die türkische Besatzung im 17. Jahrhundert gerichtete politische Schrift. Der Titel des hier zu besprechenden Werk ist also in mehrfacher Hinsicht vieldeutig. Die Irrlehren, um die es geht, werden mit einer das Volk aufwiegelnden Droge verglichen; áfium ist im Ungarischen die alte Bezeichnung für Opium.

Das Werk beginnt mit einem Beitrag des Herausgebers László Honti, der die Grundlagen der historischen Sprachwissenschaft erläutert. Er legt anschaulich dar, worauf Sprachverwandtschaft beruht, und führt zahlreiche Beispiele sowohl aus den indogermanischen als auch aus den uralischen Sprachen an. Der Beitrag ist allgemeinverständlich geschrieben, und auch ein Leser, der mit der Sprachwissenschaft nicht vertraut ist, gewinnt ohne Schwierigkeiten einen Eindruck von den Grundlagen der historisch-vergleichenden Sprachwissenschaft. Gut ein Viertel des Gesamtumfangs des vorliegenden Werks beansprucht Hontis zweiter, etwa hundert Seiten umfassender Artikel, in dem er die Geschichte der "Irrlehren“ behandelt und aus der heutigen ungarischen Debatte zum Thema zitiert.

In diesem umfangreicheren Beitrag ordnet Honti die Vertreter alternativer Auffassungen drei Kategorien zu: 1. vollständige Dilettanten ohne Grundkenntnisse in irgendeinem Fach, 2. Vertreter anderer wissenschaftlicher Fächer, Z. B. Archäologen, die willkürlich Resultate ihrer eigenen Disziplin mit der Sprachgeschichte kombinieren, und 3. Forscher, die zwar Linguisten sind, die Grundlagen der historischvergleichenden Sprachwissenschaft jedoch lückenhaft beherrschen und ihre Quellen nachlässig zitieren. Zur letzteren Kategorie zählt er u. a. die Italienerin Angela Marcantonio, die international große Aufmerksamkeit gefunden hat und deren Auffassungen in mehreren Beiträgen richtiggestellt werden. 
Gábor Bereczki und Danilo Gheno befassen sich mit denselben Themen wie Honti, indem sie die am weitesten verbreiteten Auffassungen der Hobbywissenschaftler vorstellen. Sie betrachten die postulierten Verwandtschaftsbeziehungen der Ungarn u. a. zu Hunnen, Türken, Sumerern und Etrusken, referieren die einschlägigen Behauptungen und führen Gegenargumente an. Am Ende seines Beitrags stellt Bereczki fest, dass die heutigen Ungarn es eigentlich nicht mehr nötig hätten, Verwandte unter den alten Helden- und Kulturvölkern zu suchen. Finnen und Esten als entwikkelte Nationen sollten inzwischen auch für die Ungarn akzeptable Verwandte sein!

Das Thema hat auch innenpolitische Dimensionen. Diejenigen, die alternative Theorien vorbringen, gehören häufig der nationalistischen extremen Rechten an, und besonderen Anklang finden diese Gedanken unter den ungarischen Emigranten. Béla Brogyanyi erläutert in seinem Beitrag den ideologischen und historischen Hintergrund der alternativen Theorien. Er sieht in der Suche nach einer großartigen Vergangenheit und glanzvollen Verwandten einen Versuch, das große nationale Trauma zu kurieren, das der Friedensvertrag von Trianon hinterließ. Auch der Systemwechsel 1989 hat die Verhältnisse nicht in der
Weise verbessert, wie es sich viele erhofft hatten. Die kollektive nationale Frustration verlangt zumindest eine leuchtende Vergangenheit. Die finnisch-ugrische Theorie entstand dieser Sichtweise zufolge im Auftrag der Habsburger einerseits und der Sowjetmacht andererseits, mit dem Ziel, die nationale Identität der Ungarn zu zerstören. Die Suche nach einer glanzvollen mythischen Vergangenheit ist freilich keine exklusive Angelegenheit der Ungarn; in der Türkei wird nämlich eine ähnliche Debatte geführt, und auch die dahinter stehende Ideologie ist von gleicher Art. Diese erläutert Îsmail Dogan.

Es ist schwierig, die Alternativtheoretiker von der Unrichtigkeit ihrer Auffassungen zu überzeugen, und dies, obwohl diese Auffassungen untereinander widersprüchlich sind. Man glaubt daran, weil man daran glauben will, wie Sándor Csúcs in seinem Beitrag darlegt. Er hebt hervor, dass die Finnougristen sich nicht in die Defensive begeben, sondern aktiver als bisher die wissenschaftliche Auffassung propagieren sollten. In seinem komplexen Beitrag fasst László Keresztes die Ergebnisse der finnougristischen Sprachforschung zusammen und erläutert kurz, was wir wirklich über die Sprachen und Völker wissen können, die als Verwandte der Ungarn dargestellt wurden. 
Cornelius Hasselblatt, Eberhard Winkler, László Honti und László Keresztes behandeln in ihren Beiträgen auch die alternativen Theorien, die in Finnland und Estland vorgebracht wurden und deren prominenteste Vertreter Ago Künnap und Kalevi Wiik sind. Diesen wird vorgeworfen, die Auffassungen und Ergebnisse der Archäologie und der Genetik gewaltsam mit denen der historisch-vergleichenden Sprachforschung zu verbinden. Ihrer Ansicht nach sind Genetik und Archäologie sog. harte Wissenschaften, denen die Sprachwissenschaft als Hilfswissenschaft unterzuordnen ist. Ihre Hypothese einer großen, bis an den Rhein reichenden finnischugrischen Urheimat wird in den Beiträgen widerlegt, und mit Argumenten der historisch-vergleichenden Sprachwissenschaft wird die Unmöglichkeit eines finnisch-ugrischen Substrats der germanischen und baltischen Sprachen begründet. In den vom Mainstream abweichenden Auffassungen werden zahlreiche Mängel nachgewiesen, die sowohl auf mangelnder Vertrautheit mit den Grundlagen der historischen Sprachwissenschaft als auch auf der Unkenntnis einzelner uralischer Sprachen beruhen.

Santeri Junttila behandelt in seinem Beitrag die Beziehung zwischen Unwissenschaftlichkeit und Vorgeschichte. Eines seiner Beispie- le ist die oben erwähnte Hypothese Wiiks. Zum selben Themenkreis gehört ferner der Beitrag von Merlijn de Smit. Bei einem ungarischen Leser, der nicht zur finnougrischen wissenschaftlichen Gemeinschaft gehört, werden diese Fragen jedoch kaum auf Interesse stoßen. So sind beispielsweise Wiiks Hypothesen, die in Finnland großes Medieninteresse fanden, vorwiegend auf Finnisch erschienen, dürften also in Ungarn nicht allgemein bekannt sein. Es wäre der Stringenz des vorliegenden Werks sicherlich zugute gekommen, wenn man diese Themen aus der Debatte über den Ursprung der ungarischen Sprache ausgegrenzt hätte.

Finnland vertreten neben Junttila Esa Itkonen und Johanna Laakso. Itkonen behandelt in seinem Beitrag die Kritik am Stammbaummodell. Er stellt fest, dass die Kritiker den metaphorischen Charakter dieses Modells übersehen haben und dass es weiterhin ein wesentliches Instrument der historisch-vergleichenden Sprachwissenschaft ist. Die Sprachkontakttheorien, die in letzter Zeit große Aufmerksamkeit gefunden haben, widerlegen dieses Modell nicht. Laakso ihrerseits präsentiert die mit der finnischen Sprache verknüpften Mythen, etwa die Auffassung, das Finnische sei eine Sprache ganz eigener Prägung und zudem die „älteste Sprache Europas“. Ein 
Teil dieser Mythologie ist auch aus der Debatte über die ungarische Sprache bekannt.

A nyelvrokonságról ist eine gute Einführung in die Frage, was Sprachverwandtschaft ist, was sie nicht ist und welche außerwissenschaftlichen Motive diejenigen leiten können, die für die sog. Alternativtheorien eintreten. Einem kritischen und aufgeklärten Leser bietet das Werk leicht zugängliche Informationen über die Grundlagen und die Geschichte der finnougrischen Sprachwissenschaft. Leider muss jedoch festgestellt werden, dass das Buch die Anhänger der Alternativtheorien kaum überzeugen wird. Wie bereits erwähnt, liegen ihre Motive außerhalb der Sprachwissenschaft. Zudem ist der Stil einiger Beiträge so unverblümt, dass sich wohl kein Vertreter der Gegenseite versucht fühlt, seine Auffassungen zu überprüfen. Das Buch ist außerdem viel zu umfangreich, um das Interesse des breiten Publikums $\mathrm{zu}$ wecken. Einerseits werden viele
Perspektiven angesprochen, andererseits jedoch manches mehrfach behandelt.

Den finnischen Leser wiederum verblüffen die breite Anerkennung und die Aktualität der alternativen Auffassungen in Ungarn und die Vielzahl der Publikationen zum Thema. Die Unterschiede zwischen der finnischen und der ungarischen Kultur, Gesellschaft und Mentalität erweisen sich wieder einmal als beträchtlich. Es ist schwer vorstellbar, dass man in Finnland in diesem Umfang eine öffentliche Debatte über den Ursprung der finnischen Sprache führen und dass diese Debatte Teil der politischen Rhetorik werden könnte. Wie Danielo Gheno in seinem Beitrag schreibt: „Úgy tünik, magyarnak lenni valamiféle állandó nyugtalanságot táplál.“ 'Ungar zu sein, nährt eine Art permanente Unruhe.'

Harri Mantila 